\title{
Association Between Lifestyle Characteristics and Body Mass Index of Mothers of Children With Allergic Diseases
}

\author{
Yasuko Fukuda $^{\mathrm{a}, \mathrm{c}}$, Shumi Yamamoto ${ }^{\mathrm{a}}$, Makoto Kameda ${ }^{\mathrm{b}}$
}

\begin{abstract}
Background: For mothers of children with allergic diseases, the amount of physical activity involved in childcare increases owing to factors, such as the need for diet therapy and environmental improvements. Reportedly, the body mass index (BMI) of mothers of children with food allergies (FAs) is significantly lower than that of those of children without allergies (non-FA mothers). The aim of this study was to evaluate the characteristics of diet and physical activity in FA mothers and to clarify their effects on BMI.
\end{abstract}

Methods: To investigate the association between lifestyle characteristics and BMI in 69 mothers of children with FA, bronchial asthma and atopic dermatitis, their diets and physical activity pattern (using a three-axis accelerometer) were investigated; dietary and physical activity patterns (every hour) were extracted using principal component analysis, and multiple regression analyses were performed.

Results: Multiple regression analyses revealed a significant positive correlation $(\mathrm{P}=0.037)$ between $\mathrm{BMI}$ and the third principal component of dietary patterns (positive correlation with cereals and negative correlation with sweets), a significant negative correlation $(\mathrm{P}=0.004)$ between BMI and FA and the total daily duration of performing low- and moderate-intensity physical activity $(\mathrm{P}=0.031)$ and a significant positive correlation $(\mathrm{P}=0.008)$ between FA and the first principal component of physical activity expenditure patterns (patterns of ongoing physical activity throughout the day). In FA mothers $(n=51)$, a significant positive correlation $(P=0.042)$ was observed between the third principal component of dietary patterns and BMI.

Conclusion: Low BMI in FA mothers may be related to reduced ce-

Manuscript submitted September 3, 2019, accepted September 16, 2019

aDepartment of Food Science and Nutrition, School of Human Environmental Sciences, Mukogawa Women's University, 6-46 Ikebiraki-cho, Nishinomiya, Hyogo Prefecture, 663-8558, Japan

bDepartment of Pediatrics, Osaka Habikino Medical Center, 3-7-1, Habikino, Habikino, Osaka Prefecture, 583-8588, Japan

${ }^{\mathrm{c}}$ Corresponding Author: Yasuko Fukuda, Department of Food Science and Nutrition, School of Human Environmental Sciences, Mukogawa Women's University, 6-46 Ikebiraki-cho, Nishinomiya, Hyogo Prefecture, 663-8558, Japan.

Email: yafukuda@mukogawa-u.ac.jp

doi: https://doi.org/10.14740/jocmr3976 real intake, increased sweets intake and prolonged and continuous low- and moderate-intensity physical activity.

Keywords: Body mass index; Child with allergic disease; Food allergy; Mother; Physical activity; Dietary pattern; Physical expenditure kcal pattern

\section{Introduction}

In children with food allergies (FAs) in the family (children with FA), the burden of meal preparation increases because of the need for elimination-substitution diet therapy. In addition, many children with FA experience complications of other allergic diseases, such as bronchial asthma (BA) and atopic dermatitis (AD); as a mitigation strategy, it is essential to improve the environment by removing mites and dust from the room and maintaining the cleanliness of the bedding $[1,2]$. Therefore, the amount of physical activity required for household work may increase. However, to the best of our knowledge, no studies have investigated the physical activity level of mothers of children with FA (FA mothers).

The meal content for families with children with FA has reportedly shown a tendency to be synchronized with the elimination-substitution diet to continuously provide this diet to the FA child and thus prevent mistakes and the risk of anaphylaxis (An) [3-5]. From these observations, it appears that certain characteristics are observed in the physical activities and diets of FA mothers themselves or the mothers and their families.

Currently, a physical activity meter that uses three-axis acceleration (hereinafter referred to as an activity meter) is being used to measure physical activity and accurately estimate low-intensity activities, such as the activities of daily living [6]. Moreover, to comprehensively evaluate diets, analyses based on dietary patterns that evaluate combinations between foods are widely used in various fields of research [7].

Therefore, for mothers of children with allergic diseases, a research plan was drafted to investigate the status of dietary practices and physical activities and to clarify the lifestyle characteristics that contribute to the low body mass index (BMI) of FA mothers. 


\section{Patients and Methods}

Between January 2017 and August 2018, mothers of children with allergic diseases who were being consulted at the Department of Pediatrics of Osaka Habikino Medical Center were instructed to respond to a questionnaire survey and cooperate with a measurement survey using activity meters. Written consent for the study was obtained from 106 participants. Of these, 69 (65.1\%) participants were included and 37 (34.9\%) who did not meet the eligibility criterion of being properly fitted with an activity meter were excluded. The reason for not being properly fitted with an activity meter was the insufficient duration or number of days for which the activity meter was worn.

These 69 participants included 51 mothers of children with FA (18 mothers of children with FA alone, six mothers of children with FA and BA, 18 mothers of children with FA and $\mathrm{AD}$ and nine mothers of children with FA, BA and AD), and 18 mothers of children without FA (six mothers of children with BA alone, five mothers of children with $\mathrm{AD}$ alone and seven mothers of children with BA and AD).

Exclusion criteria were defined as situations where the attending physician considered the participants inappropriate for the study, such as if the mother had any physical or psychiatric illnesses, was on medication, or if the child had a chronic illness other than allergic diseases.

\section{Profile survey: dietary survey}

Profiles were investigated using a self-administered free-form text questionnaire. The questionnaire content included the following: age, height and body weight of the mother; number of family members; family composition; age of the FA child; content of the allergy-causing food; use or non-use of substitution food; presence/absence of An experience; and presence/ absence of FA, AD and BA. All the information was self-reported; the BMI values were calculated from the height and weight values reported by the mothers themselves, and no anthropometric measurements were performed. If tofu and milk were used instead of eggs, soy milk and small fish were used instead of milk, or if rice flour and white sorghum were used instead of wheat flour, we assumed that food substitution was performed.

Diets were investigated using the brief-type self-administered diet history questionnaire (BDHQ). BDHQ estimates the energy and nutrient intake by food group based on the frequency of food consumption within a week for the previous 1 - 2 months and accordingly provides reproducibility and validity without placing a burden on the participants [8, 9]. Energy intake was calculated per $1 \mathrm{~kg}$ of body weight, and nutrient intake, other than energy, was calculated using a converted value per $1,000 \mathrm{kcal}$.

Thereafter, dietary patterns were extracted using principal component analysis based on the intake of 15 food groups from the results of the dietary survey. Criteria for extracting the principal component factors were set at a characteristic value of $\geq 1$, and the food group indicating the principal com- ponent was set at a factor load of $\geq 0.30$.

\section{Physical activity survey}

Status of physical activity was measured using an activity meter. A three-axis accelerometer (OMRON HJA-750C) was used as the activity meter. This device has previously been tested for its reliability and validity in measuring low- and moderate-intensity activities of daily living other than exercise, such as housework and walking [6-13]. Examples of low-intensity (1.5 - 2.9 metabolic equivalents (METs)) activities are bathing and having conversations while standing, those of moderate-intensity (3.0 - 5.9 METs) activities include childcare (while standing) and vacuuming and those of highintensity ( $\geq 6$ METs) activities include climbing stairs at a slightly faster pace [14].

Participants who provided consent were asked to wear an activity meter. The participant was required to wear the activity meter in front of the lumbar region for at least $10 \mathrm{~h}$ daily and for at least 7 consecutive days (including Saturdays and Sundays); the participant must wear the meter upon waking up and continue wearing it till bedtime, with the exception of situations when it could not be worn, such as during baths. The meters were collected on the day of the next visit. Data extracted from the activity meters were considered valid if the meter was worn for at least $600 \mathrm{~min}$, and data were obtained for 7 - 10 consecutive days.

The major components of total daily energy expenditure were basal metabolism, physical activity and diet-induced thermogenesis. Total daily energy consumption and basal metabolism were converted to per $1 \mathrm{~kg}$ of body weight. Physical activities included activities of daily living other than walking and walking that were expressed as a percentage of the total daily energy expenditure in terms of calories burned while walking and calories burned while performing daily living activities. In addition, the duration of total daily activity (min/day) was calculated for each activity intensity: high, medium and low.

Thereafter, the energy spent for performing high-, medium- and low-intensity activities was summed up at hourly intervals between 6 o'clock and 24 o'clock, and principal component analyses were used to extract the patterns of physical expenditure in kcal. Criteria for extracting the principal component factors were set at a characteristic value of $\geq 1$, and the time zone to signify the principal component was set at a factor load of $\geq 0.30$.

\section{Multiple regression analyses}

Multiple regression analyses were performed. To investigate the association between BMI and diet, BMI was used as an objective variable and six explanatory variables, including the age of the mother and child and the principal components of dietary patterns with characteristic values of $\geq 1$, were used. Moreover, to confirm the association between the third principal component of dietary pattern and BMI in 51 FA mothers, the third principal component of dietary pattern was introduced as the objective var- 
iable; the age and BMI of the mother, three major allergy causes in the child, substitute food use and the presence or absence of An experience were introduced as explanatory variables.

To investigate the association between BMI and status of physical activity, BMI was used as the objective variable; the age of the mother and child, presence or absence of FA, AD and $\mathrm{BA}, \%$ calories burned on walking activities, $\%$ calories burned while performing activities of daily living and the total daily duration of low- and medium-intensity activities were used as explanatory variables. To confirm the association between allergic diseases and physical expenditure kcal patterns, the presence or absence of $\mathrm{FA} / \mathrm{BA} / \mathrm{AD}$ was used as an objective variable, and the five principal components of physical expenditure kcal patterns with characteristic values of $\geq 1$ as well as the ages of FA mothers and children were used as the explanatory variables.

\section{Statistical analyses}

Statistical Analysis System (SAS) version 9.4 (SAS Institute Inc., Cary, NC, USA) was used for statistical analyses. For descriptive statistics, mean \pm standard deviation (SD) is shown unless specified otherwise. Categorical variables were compared between the two groups using the Fisher's exact test. Principal component analysis was used to extract dietary patterns and physical expenditure kcal patterns. Multiple regression analysis (stepwise) was used to test linear regressions. P $<0.05$ on twotailed testing was considered as a significant difference.

\section{Ethical considerations}

The present study was conducted with the approval of the ethical review board of Osaka Habikino Medical Center (approval no.: 777-2, August 24, 2016), the ethical review board for research of Mukogawa Women's University, Junior College Division (approval no.: No. 16-87, December 17, 2016) and was performed in accordance with the Declaration of Helsinki. The study was conducted after explaining its details to the study participants in advance and obtaining written consent from them. For the use in the studies that need consideration, information was used with due care with the consent of the participants and handled in an anonymized fashion to ensure that individuals could not be identified.

\section{Results}

\section{Profile survey}

The profile of subjects is shown in Table 1. Data pertaining to the smoking status were not collected.

\section{Dietary patterns}

Nutrient intake data are presented in Table 2. Six principal
Table 1. Participant Characteristics

\begin{tabular}{ll}
\hline Characteristics & \\
\hline Overall & 69 \\
$\quad$ Number of mothers & $39.9 \pm 4.2$ \\
Age of mothers (years) & $159.9 \pm 5.4$ \\
Height of mothers (cm) & $53.1 \pm 7.9$ \\
Body weight of mothers $(\mathrm{kg})$ & $20.8 \pm 3.1$ \\
Body mass index of mothers $\left(\mathrm{kg} / \mathrm{m}^{2}\right)$ & $4.2 \pm 0.8$ \\
Number of families & $7.5 \pm 3.2$ \\
Age of children (years) & \\
Children's characteristics & $28 / 41$ \\
Bronchial asthma (presence/absence) & $39 / 30$ \\
Atopic dermatitis (presence/absence) & $51 / 18$ \\
Food allergy (presence/absence) & $23 / 46$ \\
Substitute food use (use/not use) & $29 / 40$ \\
Anaphylaxis (presence/absence) & \\
\hline
\end{tabular}

Data are expressed as means \pm standard deviation.

components with characteristic values of $\geq 1$ were extracted. Six dietary patterns accounted for $72.4 \%$ of the total variance. Food groups with a factor load of $\geq 0.30$ were selected, and the content was interpreted according to principal components. The factor loads up to the sixth principal component are shown in Table 3.

Green and yellow vegetables $(-0.501)$, other vegetables $(-0.441)$, legumes $(-0.394)$ and fishes $(-0.355)$ were extracted in the negative direction for the first principal component; therefore, these were interpreted as "non-substitute food type". Meat (0.475), beverages other than alcohol (0.429), oils $(0.380)$ and sugars $(0.324)$ were extracted in the positive direction and cereals $(-0.313)$ were extracted in the negative direction, which was interpreted as "partial side dish type", for the second principal component. Cereals (0.584) and seasonings $(0.391)$ were extracted in the positive direction for the third principal component, and sweets $(-0.512)$ were extracted in the negative direction, which was interpreted as "ordinary type". From the fourth principal component onward, it was interpreted as an elimination-substitution diet in which a specific food group was removed, because there was an association between milk and eggs and oils and fats.

\section{Physical activity-physical expenditure kcal patterns}

The status of physical activity is shown in Table 4. Five principal components with a characteristic value of $\geq 1$ were extracted for the physical expenditure kcal patterns. The five physical expenditure kcal patterns accounted for $80.6 \%$ of the total variance. Factor load of $>0.30$ was selected, and the content was interpreted for each principal component. The factor loads up to the fifth principal component are shown in Table 5 .

The first principal component was interpreted as "ordinary 
Table 2. Nutritional Intake

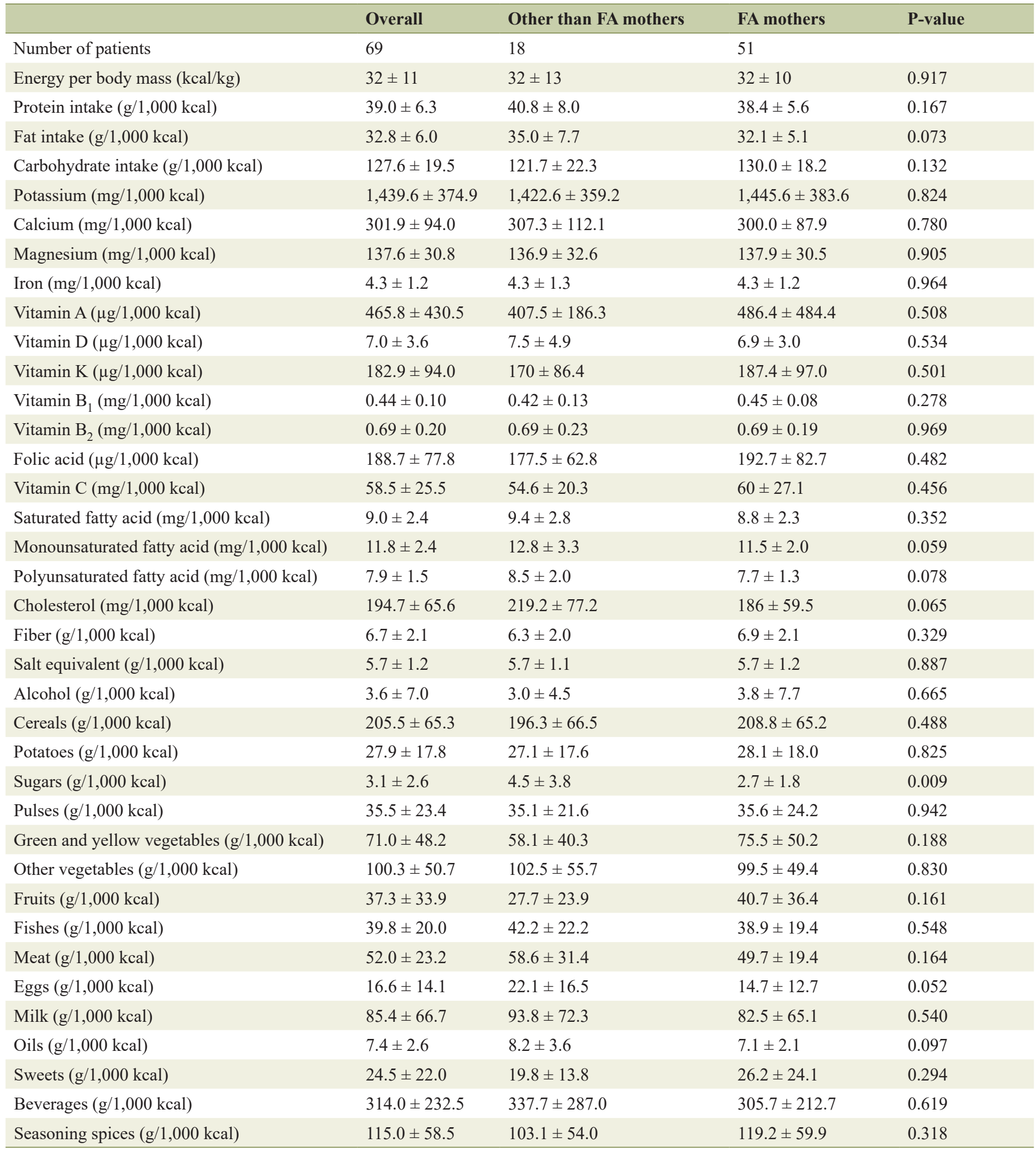

Data are expressed as mean \pm standard deviation. Mann-Whitney $U$ test as appropriate. FA: food allergy.

type", because there was no time period in which there was an association. The second principal component was interpreted as "night type" because of a positive weight from 20 o'clock to 24 o'clock, and the second principal component was inter- 
Table 3. Factor Loading of Each Food Group by Factor Analysis

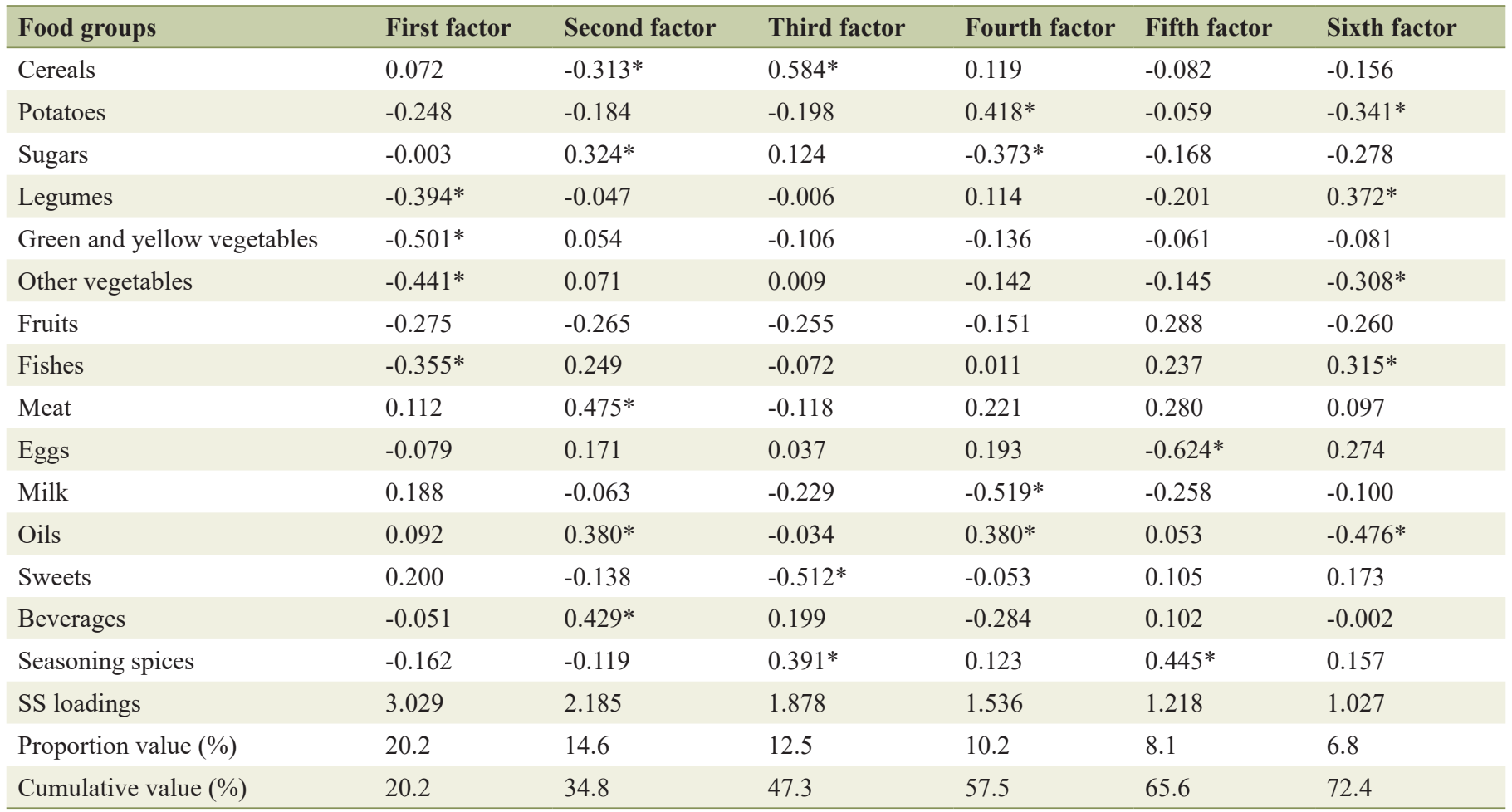

*: $\mid$ load $\mid>0.30$ (in module).

preted as "non-morning type" because of a negative weight from 6 o'clock to 9 o'clock (Table 5).

\section{Multiple regression analysis}

The associations between BMI and diet and status of physical activity are shown in Table 6. The status of diet was as follows: there was a significant positive correlation between BMI and the third principal component of dietary patterns $(\mathrm{P}=0.037)$. A significant positive correlation between the third principal component of dietary patterns and BMI was confirmed for FA mothers $(\mathrm{n}=51 ; \mathrm{P}=0.042)$ (Table 7$)$. No association was observed between the third principal component and BMI in nonFA mothers $(n=18)$.

In physical activity status, there was a significant negative

Table 4. Results of the Physical Activity Level Survey

\begin{tabular}{ll}
\hline & Overall (n=69) \\
\hline Basic metabolic rate (kcal/day) & $1,139 \pm 104$ \\
Basic metabolic rate/body weight (kcal/kg) & $21.6 \pm 1.4$ \\
Total energy spent per day (kcal/day) & $2,003 \pm 176$ \\
Total energy spent per day/body weight (kcal/kg) & $38.1 \pm 3.7$ \\
Physical activity level & $1.76 \pm 0.11$ \\
No. of steps (steps/day) & $7,208 \pm 2,067$ \\
Calories burned by walking (\%) & $9.3 \pm 2.8$ \\
Calories burned by activities of daily living (\%) & $23.7 \pm 4.1$ \\
Duration of high-intensity ( $\geq 6 \mathrm{METs})$ physical activity (min/day) & $3.3 \pm 2.8$ \\
Duration of moderate-intensity (3.0 - 5.9 METs) physical activity (min/day) & $99 \pm 24.2$ \\
Duration of low-intensity (1.5 - 2.9 METs) physical activity (min/day) & $355.8 \pm 73.3$ \\
\hline
\end{tabular}

Data are expressed as mean \pm standard deviation. ${ }^{a}$ Physical activity level $=$ total energy spent per day (kcal/day)/basic metabolic rate $(\mathrm{kcal} / \mathrm{day})$. ${ }^{\mathrm{b} C}$ Calories burned by walking $=$ total calories burned by walking (kcal/day)/total energy spent per day (kcal/day) $\times 100 .{ }^{\circ}$ Calories burned by activities of daily living $=$ total calories burned by activities of daily living (kcal/day)/total energy spent per day (kcal/day) $\times 100$. METs: metabolic equivalents. 
Table 5. Physical Expenditure Kcal Patterns

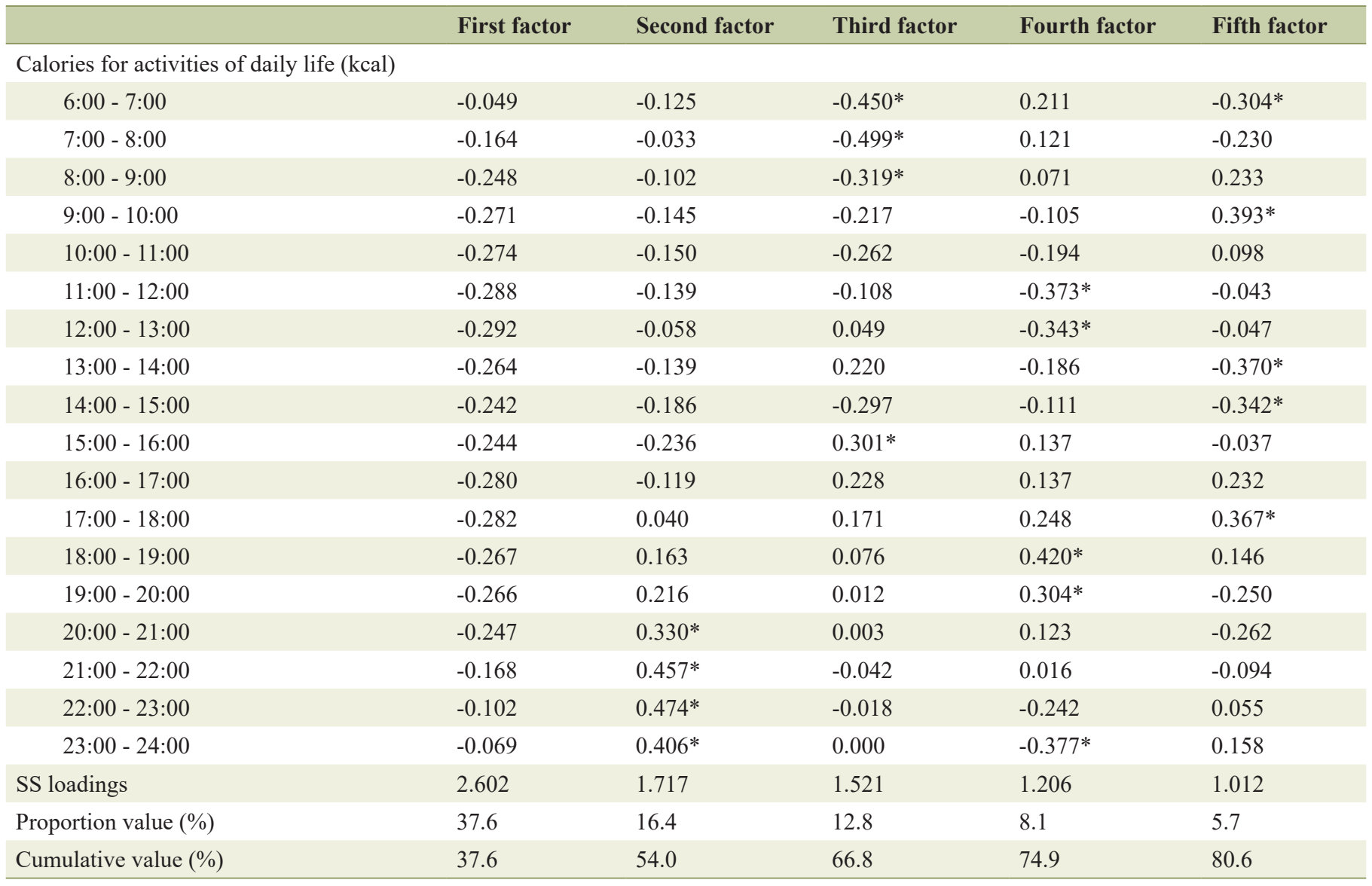

*: $\mid$ load $\mid>0.30$ (in module).

correlation between BMI and the presence or absence of FAs $(\mathrm{P}=0.004)$ and a significant negative correlation between BMI and the total daily activity time of low- and medium-intensity activities $(\mathrm{P}=0.031)$ (Table 6).

Relationships between allergic diseases and physical expenditure kcal patterns are shown in Table 8. There was a significant positive correlation between FA and the first principal component, which confirmed that the lifestyle characteristics of physical activity were always performed between waking and bedtime.

\section{Discussion}

The aim of the present study was to investigate the daily diets and status of physical activity in mothers of children with at

Table 6. Multiple Regression Models of BMI

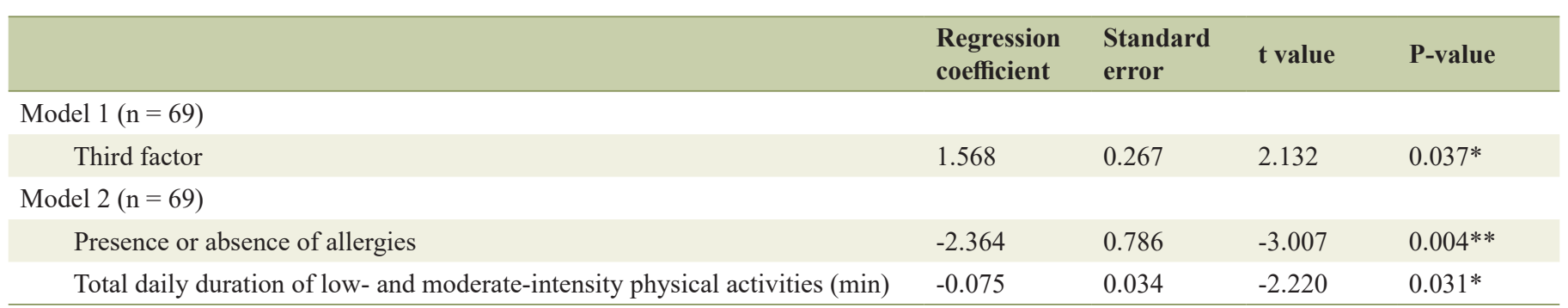

${ }^{*} \mathrm{P}<0.05,{ }^{* *} \mathrm{P}<0.01$. Stepwise multiple regression analysis. Model 1: BMI was used as an objective variable, and the mother's and child's ages and six principal components from dietary patterns with characteristic values $\geq 1$ were used as explanatory variables. Model 2: BMI was used as an objective variable, and the explanatory variables were the mother's and child's ages, presence or absence of FA, BA, or AD, \% calories burned while walking, \% calories burned while performing ADLs and total daily duration of low- and moderate-intensity activities. BMI: body mass index; FA: food allergy; BA: bronchial asthma; AD: atopic dermatitis; ADL: activities of daily living. 
Table 7. Association Between Dietary Patterns (Third Principal Component Factor) and Food Allergies/Profile

\begin{tabular}{|c|c|c|c|c|}
\hline & Regression coefficient & Standard error & t value & P-value \\
\hline Age of mothers & -0.045 & 0.052 & -0.856 & 0.397 \\
\hline BMI of mothers & 0.145 & 0.069 & 2.094 & $0.042 *$ \\
\hline Substitute food use & -0.173 & 0.432 & -0.400 & 0.691 \\
\hline Presence or absence of anaphylaxis & -0.837 & 0.402 & -2.083 & $0.043 *$ \\
\hline
\end{tabular}

${ }^{*} P<0.05, n=51$. The objective variable was the third principal component in dietary patterns, and the explanatory variables were the mother's age and BMI, the three major causes of food allergy in the child, use of substitute foods, and presence or absence of An experience. BMI: body mass index.

least one of FA, AD and BA or multiple concomitant allergic diseases for evaluating the association between lifestyle characteristics of the mother and BMI and, in particular, for clarifying the lifestyle characteristics that affect low BMI observed in FA mothers.

The results showed that the BMI of FA mothers may be related to dietary biases, such as low cereal consumption and high sweet consumption, and physical activity, such as low and moderate physical activity over a long period of time and continuous activity.

Regarding the effect of diets, dietary patterns were extracted, and the association with BMI was evaluated. A positive correlation was observed between BMI and the third principal component. In addition, a significant positive correlation between the third principal component and BMI was confirmed for FA mothers. However, no association was observed in nonFA mothers. These findings suggest that low BMI in FA mothers is related to lower cereal consumption and higher sweet consumption and that this pattern of consumption of such foods is limited to FA mothers. When the consumption of a common type of meal, such as a staple dish with a side dish, was difficult, it was considered that the shortage in content would be complemented by sweets. Sweets were grain-based confectioneries (e.g. rice confectioneries) that did not contain eggs or milk. Inappropriate content in diets can lead to deficiencies or biases in energy and nutrients as well as to imbalances in energy-producing nutrients. In addition, the synthesis of muscle proteins requires a certain amount of protein per meal [15]. These results suggest that dietary and nutritional imbalances affect the maintenance of body size and muscle mass in FA mothers with low BMI.

With regard to the effect of physical activity, the association with BMI was evaluated by focusing on the type of allergic diseases, total daily duration of low- and mediumintensity activities, and physical expenditure kcal patterns. Significant negative correlations were observed between BMI and FA as well as between BMI and the total daily duration of low- and moderate-intensity activities; moreover, there was a significant positive correlation between FA and the first principal component of physical expenditure kcal patterns. These findings suggested that the work associated with environmental improvement was added to the work associated with providing diet therapy to children with FA and that this work was continuously performed during the daytime. These results suggest that low- and moderate-intensity continuous physical activities are involved in the BMI of FA mothers.

Physical activity required to provide elimination-substitution diets and to comply with environmental improvements are categorized as low- and moderate-intensity activities. Energy expenditure is related to activity intensity and duration [16]. Evaluating the association between the intensity of activity and weight loss using a three-axis accelerometer reported that weight loss was related to the duration of activity of $>3$ and $>$ 4 METs rather than total daily steps. As a mechanism of weight loss, the proportions of carbohydrates and fats used as energy sources during physical activity have been shown to vary with activity intensity [17-19]. Lower activity increases the rate of

Table 8. Association Between Allergic Diseases and Physical Expenditure Kcal Patterns

\begin{tabular}{|c|c|c|c|c|}
\hline & Regression coefficient & Standard error & t value & P-value \\
\hline \multicolumn{5}{|l|}{ FA } \\
\hline First factor & 0.053 & 0.019 & 2.742 & $0.008 * *$ \\
\hline \multicolumn{5}{|l|}{$\mathrm{BA}$} \\
\hline Third factor & -0.082 & 0.038 & -2.161 & $0.034^{*}$ \\
\hline \multicolumn{5}{|l|}{$\mathrm{AD}$} \\
\hline
\end{tabular}

${ }^{*} \mathrm{P}<0.05$, ${ }^{* *} \mathrm{P}<0.01$. Stepwise multiple regression analysis. The objective variable was the presence or absence of $F A, B A$, or $A D$, and the explanatory variables were the mother's and child's ages and the five principal components of physical expenditure kcal patterns with characteristic values $\geq 1$. FA: food allergies; BA: bronchial asthma; AD: atopic dermatitis. 
fat used and higher activity increases the rate of carbohydrate used [20]. However, hypermetabolism persists after activities to restore the body and following a high-intensity activity, more fat is mobilized as an energy source to replace the carbohydrates supplied to the liver and muscle during activity. Women typically have a higher percentage of body fat than men, and the percentage of fat-derived energy during exercise is higher in women than in men [19]. In the present study population, children have one or more of either FA, AD, or BA. Therefore, activities of daily living related to elimination-substitution diets, such as preparation and cooking, and indoor environmental improvements, which includes low- and moderate-intensity physical activities, may be performed throughout the day. By extracting physical expenditure kcal patterns and evaluating the association with low- and medium-intensity physical activities, these physical activities were found to be continuously performed during the time the FA mothers were awake. Therefore, a significant negative correlation between BMI and FA as well as a significant negative correlation between BMI and the total daily duration of low- and moderate-intensity activities were considered valid results.

In addition to the naturally occurring long-term health effects of weight loss and decrease in BMI in women, it has been shown that pregnancy affects the health of the subsequent generation [21-23]. Considering that mothers of children with FA are in a generation that can become pregnant, the necessity for nutrition support, which is a point that has been overlooked until now, for children with FA as well as FA mothers was revealed.

The strength of the present study is that it is the first instance of clarification of lifestyle characteristics of FA mothers. The aim of this study was to clarify whether diet biases and increases in and continuation of low- and moderate-intensity activities in relation to providing diet therapy for children with FA are involved in the BMI of FA mothers.

\section{Study limitations}

The present study has several limitations that ought to be considered. First, this was a cross-sectional study that cannot prove causality. Second, the multivariate analyses did not consider confounding factors, such as socioeconomic status and the impact of exercise habits. Third, the height and weight of mothers and answers to mother and child dietary surveys were self-reported by the mothers themselves and there may be recall bias. In addition, the effects of housework and lifestyle activities on the care of children with FA are not limited to mothers and may affect the brothers and sisters of children with FA, their fathers, and family members living in the same household; however, these effects have not been investigated in the present study. Therefore, the possibility of some bias must be considered.

\section{Conclusions}

Multiple regression analyses were performed using the BMI of mothers of children with allergic diseases as an objective variable and factors related to dietary habits and physical activity as explanatory variables. Regarding the effect of diets, there was a positive correlation with the third principal component (ordinary type: positive correlation with cereals and negative correlation with sweets) and regarding the status of physical activity, there was a negative correlation with the total daily duration of low- and moderate-intensity activity. In FA mothers, a significant positive correlation was confirmed between the third principal component of dietary patterns and BMI as well as between FA and the first principal component of physical expenditure kcal patterns (ordinary type: always active). Based on these results, the low BMI observed in FA mothers was attributable to dietary and nutrition biases as well as the duration and continuation of low- and moderate-intensity physical activity. The duration of low- and moderate-intensity physical activities is considered as the time required to perform tasks related to the provision of elimination-substitution diets and environmental improvement. In the future, it is necessary to evaluate the effects of children with FA on their brothers and sisters, fathers, and other family members living in the same household.

\section{Acknowledgments}

The authors express their sincere appreciation to the parents who regularly visited the Department of Pediatrics, Osaka Habikino Medical Center, and consented to participate in this study. Further, the authors thank Risa Okamoto, a registered dietitian who provided assistance in the analyses involving the activity meter.

\section{Financial Disclosure}

This work was supported by JSPS KAKENHI (grant number JP17K00950).

\section{Conflict of Interest}

We declare that we have no conflict of interest.

\section{Informed Consent}

The contents of the study were explained to the subjects in advance and written informed consent was obtained.

\section{Author Contributions}

Yasuko Fukuda drew up the research plan, dealt with ethical research screening, provided detailed explanations of the research to the participants, collected/analyzed the data and prepared the initial draft. Shumi Yamamoto analyzed the data. Makoto Kameda drew up the research plan, dealt with the ethi- 
cal research screening, explained the research to the participants and obtained their consent.

\section{References}

1. Ebisawa M, Ito K, Fujisawa T, Committee for Japanese Pediatric Guideline for Food Allergy TJSoPA, Clinical Immunology TJSoA. Japanese guidelines for food allergy 2017. Allergol Int. 2017;66(2):248-264.

2. Allen KJ, Dharmage SC. The role of food allergy in the atopic march. Clin Exp Allergy. 2010;40(10):1439-1441.

3. Fukuda Y, Yamamoto S, Nishida K, Takaoka Y, Kameda M. A study on the effect of nutrient intake on the body mass index of mothers of children with food allergies. Asian Pac J Allergy Immunol. 2019.

4. Allen CW, Bidarkar MS, vanNunen SA, Campbell DE. Factors impacting parental burden in food-allergic children. J Paediatr Child Health. 2015;51(7):696-698.

5. Knibb RC, Barnes C, Stalker C. Parental self-efficacy in managing food allergy and mental health predicts food allergy-related quality of life. Pediatr Allergy Immunol. 2016;27(5):459-464.

6. Oshima Y, Kawaguchi K, Tanaka S, Ohkawara K, Hikihara Y, Ishikawa-Takata K, Tabata I. Classifying household and locomotive activities using a triaxial accelerometer. Gait Posture. 2010;31(3):370-374.

7. Kant AK. Dietary patterns and health outcomes. J Am Diet Assoc. 2004;104(4):615-635.

8. Kobayashi S, Murakami K, Sasaki S, Okubo H, Hirota N, Notsu A, Fukui M, et al. Comparison of relative validity of food group intakes estimated by comprehensive and brief-type self-administered diet history questionnaires against $16 \mathrm{~d}$ dietary records in Japanese adults. Public Health Nutr. 2011;14(7):1200-1211.

9. Kobayashi S, Honda S, Murakami K, Sasaki S, Okubo H, Hirota N, Notsu A, et al. Both comprehensive and brief self-administered diet history questionnaires satisfactorily rank nutrient intakes in Japanese adults. J Epidemiol. 2012;22(2):151-159.

10. Bassett DR, Jr., Ainsworth BE, Swartz AM, Strath SJ, O'Brien WL, King GA. Validity of four motion sensors in measuring moderate intensity physical activity. Med Sci Sports Exerc. 2000;32(9 Suppl):S471-480.

11. Baque E, Sakzewski L, Trost SG, Boyd RN, Barber L. Validity of accelerometry to measure physical activity intensity in children with an acquired brain injury. Pediatr
Phys Ther. 2017;29(4):322-329.

12. Dlugonski D, DuBose KD, Rider P. Accelerometer-measured patterns of shared physical activity among motheryoung child dyads. J Phys Act Health. 2017;14(10):808814.

13. Hnatiuk JA, Ridgers ND, Salmon J, Hesketh KD. Maternal correlates of young children's physical activity across periods of the day. J Sci Med Sport. 2017;20(2):178-183.

14. Ainsworth BE, Haskell WL, Whitt MC, Irwin ML, Swartz AM, Strath SJ, O'Brien WL, et al. Compendium of physical activities: an update of activity codes and MET intensities. Med Sci Sports Exerc. 2000;32(9 Suppl):S498-504.

15. Phillips SM. A brief review of critical processes in exercise-induced muscular hypertrophy. Sports Med. 2014;44(Suppl 1):S71-77.

16. Hara T, Matsumura Y, Yamamoto M, Kitada T, Nakao H, Nakao H, Suzuki T, et al. The relationship between body weight reduction and intensity of daily physical activities assessed with 3-dimensional accelerometer. Jpn J Phys Fit Sport. 2006;55(4):385-392.

17. Wolfe RR, Klein S, Carraro F, Weber JM. Role of triglyceride-fatty acid cycle in controlling fat metabolism in humans during and after exercise. Am J Physiol. 1990;258(2 Pt 1):E382-389.

18. Spriet LL. New insights into the interaction of carbohydrate and fat metabolism during exercise. Sports Med. 2014;44(Suppl 1):S87-96.

19. Blaak E. Gender differences in fat metabolism. Curr Opin Clin Nutr Metab Care. 2001;4(6):499-502.

20. Yoshioka T, Ogasawara K. On the metabolism of carbohydrates and lipids during muscular Exercise (I) changes in the concentration of blood sugar and plasma free fatty acids during long distance running. Jpn J Phys Educ Hlth Sport Sci. 1972;17(3):143-150.

21. Han Z, Mulla S, Beyene J, Liao G, McDonald SD, Knowledge Synthesis G. Maternal underweight and the risk of preterm birth and low birth weight: a systematic review and meta-analyses. Int J Epidemiol. 2011;40(1):65-101.

22. Rahman MM, Abe SK, Kanda M, Narita S, Rahman MS, Bilano V, Ota E, et al. Maternal body mass index and risk of birth and maternal health outcomes in low- and middle-income countries: a systematic review and metaanalysis. Obes Rev. 2015;16(9):758-770.

23. Liu P, Xu L, Wang Y, Zhang Y, Du Y, Sun Y, Wang Z. Association between perinatal outcomes and maternal prepregnancy body mass index. Obes Rev. 2016;17(11):10911102. 